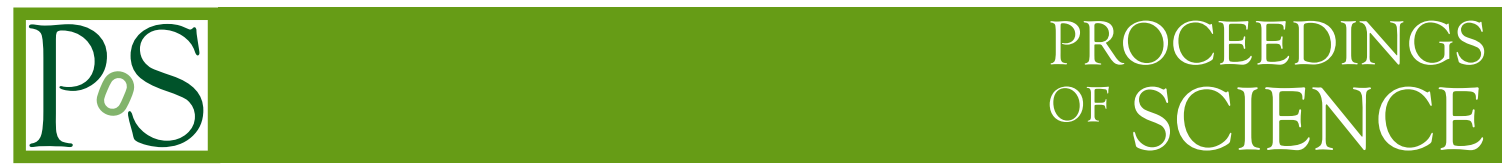

\title{
Definition and Evolution of GTMDs
}

\section{Miguel G. Echevarria*}

Departament de Física Quàntica i Astrofísica and Institut de Ciències del Cosmos, Universitat de Barcelona, Martí i Franquès 1, 08028 Barcelona, Spain

E-mail: mgechevarria@fqa.ub.edu

Generalized transverse momentum distributions, one of the most fundamental hadronic quantities, share the same problematic issue as the transverse momentum distributions: rapidity divergences. In order to cancel them, a (square root of a) soft function should also be included in their definition. By doing this, we manage to obtain their evolution kernel, which is universal for all polarizations.

QCD Evolution 2016

May 30-June 03, 2016

National Institute for Subatomic Physics (Nikhef), Amsterdam

* Speaker. 
In [1] we argue that the hadronic matrix elements called GTMDs, as currently formulated and analyzed in the literature [2, 3, 4], are improperly defined. This results from two observations: on one hand, these matrix elements lack proper evolution properties with respect to the renormalization scale $\mu$ and the rapidity scale $Q$; and on the other, their operator product expansion into generalized parton distributions breaks down even for large enough transverse momentum, contrary to what it should be.

When calculated perturbatively, as we show below for an unpolarized quark target, the existing definition of GTMDs suffers from unwanted spurious rapidity divergences (RDs). Their existence is not surprising: in fact, the situation is similar to the case of the by-now familiar TMDs [5, $6,7,8]$. The fact that the GTMD correlator is off-diagonal in hadron momenta only makes the QCD corrections more laborious to obtain, however the fundamental observation regarding the appearance of spurious RDs remains the same.

At the operator level ${ }^{1}$, the already existing definition of leading-twist quark GTMDs is given by [3]

$$
\phi_{\lambda \lambda^{\prime}}^{[\Gamma], q}=\left.\frac{1}{2}\left\langle p^{\prime}, \lambda^{\prime}\left|\bar{q}(-z / 2) \mathscr{W}_{n}(-z / 2) \Gamma \mathscr{W}_{n}^{\dagger}(z / 2) q(z / 2)\right| p, \lambda\right\rangle\right|_{z^{+}=0},
$$

where $\lambda, \lambda^{\prime}$ are the nucleon helicities and the matrix $\Gamma=\left\{\gamma^{+}, \gamma^{+} \gamma_{5}, i \sigma^{j+} \gamma_{5}\right\}$ stands for an unpolarized, longitudinally polarized or transversely polarized quark, respectively. Gauge invariance among regular gauges is satisfied by the inclusion of the collinear gauge link or Wilson line $\mathscr{W}_{n}$. This Wilson line can be past-pointing or future-pointing [4], but for definiteness we choose the one consistent with DIS kinematics:

$$
\mathscr{W}_{n ; \alpha \beta}(z)=\left\{\mathscr{P} \exp \left[-i g \int_{0}^{\infty} d s \bar{n} \cdot A(z+s \bar{n})\right]\right\}_{\alpha \beta},
$$

where the gluon field $A$ stands for collinear gluon field in the $n$ direction. To ensure gauge invariance among singular and regular gauges, one needs to also introduce transverse gauge links at light-cone infinities $\left(z^{-}=\infty\right)[9,10,11]$.

Motivated by the proper treatment of TMDs $[5,6,7,8]$, we arrive at the following result for the properly defined quark GTMDs:

$$
W_{\lambda \lambda^{\prime}}^{[\Gamma], q}=\frac{1}{2} \int \frac{d z^{-} d^{2} z_{\perp}}{(2 \pi)^{3}} e^{+i\left(\frac{1}{2} z^{-} \bar{k}^{+}-z_{\perp} \cdot \bar{k}_{\perp}\right)} \phi_{\lambda \lambda^{\prime}}^{[\Gamma], q}\left(0, z^{-}, z_{\perp}\right) S^{\frac{1}{2}}\left(z_{T}\right),
$$

where the soft function

$$
S\left(z_{T}\right)=\left.\frac{\operatorname{Tr}_{c}}{N_{c}}\left\langle 0\left|\mathscr{S}_{n}^{\dagger}\left(-\frac{z}{2}\right) \mathscr{S}_{\bar{n}}\left(-\frac{z}{2}\right) \mathscr{S}_{\bar{n}}^{\dagger}\left(\frac{z}{2}\right) \mathscr{S}_{n}\left(\frac{z}{2}\right)\right| 0\right\rangle\right|_{z^{ \pm}=0}
$$

with the following soft Wilson lines in DIS kinematics:

$\mathscr{S}_{n ; \alpha \beta}(z)=\left\{\mathscr{P} \exp \left[i g \int_{-\infty}^{0} d s n \cdot A(z+s n)\right]\right\}_{\alpha \beta}, \quad \mathscr{S}_{\bar{n} ; \alpha \beta}(z)=\left\{\mathscr{P} \exp \left[-i g \int_{0}^{\infty} d s \bar{n} \cdot A(z+s \bar{n})\right]\right\}_{\alpha \beta}$.

\footnotetext{
${ }^{1} \mathrm{~A}$ generic vector $v^{\mu}$ is decomposed as $v^{\mu}=v^{+} \frac{n^{\mu}}{2}+v^{-\frac{n^{\mu}}{2}}+v_{\perp}^{\mu}=\left(v^{+}, v^{-}, v_{\perp}\right)$, where $v^{+} \equiv \bar{n} \cdot v$ and $v^{-} \equiv n \cdot v$, with the light-cone vectors defined by $n=(1,0,0,1)$ and $\bar{n}=(1,0,0,-1)$. We also use $v_{T} \equiv\left|v_{\perp}\right|$, so that $v_{T}^{2}=v_{\perp}^{2}=-v_{\perp}^{2}>0$.
} 


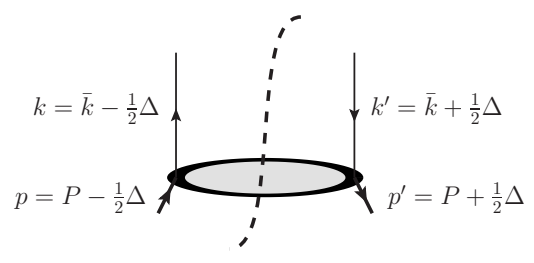

Figure 1: Kinematics for GTMDs in the symmetric frame.

This soft function has been shown to be universal $[6,7,12,13]$.

Couple of remarks are in order. First, the given new definition of quark GTMDs can be straightforwardly extended to the case of gluon GTMDs. And second, the collinear contribution $\phi$ is implicitly understood to be the pure collinear contribution, free from soft contamination $[12,14]$.

For TMDs (not GTMDs), the factor $\sqrt{S}$ results from the splitting of the soft function contribution $S$ among two TMD correlators, where $S$ and the correlators appear in a factorization statement for a given process. For GTMDs the situation is quite different, since there is not, so far, any (factorized) process to rely on. Actually, it is a very important question if/how GTMDs can be related to experimental observables. The importance of properly defining quark and gluon GTMDs, extracting their evolution kernels, motivating their lattice calculation and experimental measurements, relies not only of them being fundamental objects of QCD, but also on their connection to orbital angular momentum of partons inside hadrons [15, 16, 17, 18, 19, 20, 21, 22, 23].

\section{NLO calculation: cancellation of rapidity divergences}

For the perturbative calculation of the GTMDs for an unpolarized quark target, we use the $\delta$ regulator (see for instance [6]) to regularize rapidity and infrared (IR) divergences, and dimensional regularization in $\overline{\mathrm{MS}}$-scheme $\left(\mu^{2} \rightarrow \mu^{2} e^{\gamma_{E}} /(4 \pi)\right)$ for ultraviolet (UV) ones. All the results below were obtained using Feynman gauge.

It is convenient to work in a symmetric frame, Fig. 1, with the average nucleon momentum $P=$ $\frac{1}{2}\left(p^{\prime}+p\right)$, the momentum transfer $\Delta=p^{\prime}-p=k^{\prime}-k$ and the average quark momentum $\bar{k}=\frac{1}{2}\left(k^{\prime}+\right.$ $k)$. These momenta are parametrized as $P^{\mu}=\left(P^{+}, P^{-}, 0_{\perp}\right)$ and $\Delta^{\mu}=\left(-2 \xi P^{+}, 2 \xi P^{-}, \Delta_{\perp}\right)$, with $P^{-}=\frac{\Delta_{T}^{2}+4 M^{2}}{4\left(1-\xi^{2}\right) P^{+}}$and $M$ being the nucleon mass. We work in a frame in which $p$ and $p^{\prime}$ have very large plus components. The GTMDs $W_{\lambda \lambda^{\prime}}^{[\Gamma], q}$ depend on the kinematical variables $\left(x, \xi, \bar{k}_{T}^{2}, \Delta_{T}^{2}, \bar{k}_{\perp}\right.$. $\left.\Delta_{\perp}\right)$, with $x=\bar{k}^{+} / P^{+}$and $\xi=-\Delta^{+} /\left(2 P^{+}\right)$. They are also functions of the renormalization and rapidity scales, as we discuss below, and our NLO results are valid in the DGLAP region, i.e. $|\xi|<x$.

The GTMD $W_{\lambda \lambda^{\prime}}^{\left[\gamma^{+}\right], q}$ can be decomposed as follows [3]

$$
W_{\lambda \lambda^{\prime}}^{\left[\gamma^{+}\right], q}=\Gamma_{1} F_{1,1}^{q}+\Gamma_{2} F_{1,2}^{q}+\Gamma_{3} F_{1,3}^{q}+\Gamma_{4} F_{1,4}^{q},
$$


where the $F_{1, i}^{q}$ functions are in general complex-valued and the Dirac helicity structures $\Gamma_{i}$ are ${ }^{2}$

$$
\begin{array}{ll}
\Gamma_{1}=\frac{1}{2 M} \bar{u}\left(p^{\prime}, \lambda^{\prime}\right) u(p, \lambda), & \Gamma_{2}=\frac{1}{2 M} \frac{\bar{k}_{\perp}^{i}}{P^{+}} \bar{u}\left(p^{\prime}, \lambda^{\prime}\right) i \sigma^{i+} u(p, \lambda), \\
\Gamma_{3}=\frac{1}{2 M} \frac{\Delta_{\perp}^{i}}{P^{+}} \bar{u}\left(p^{\prime}, \lambda^{\prime}\right) i \sigma^{i+} u(p, \lambda), & \Gamma_{4}=\frac{1}{2 M} \frac{\bar{k}_{\perp}^{i} \Delta_{\perp}^{j}}{M^{2}} \bar{u}\left(p^{\prime}, \lambda^{\prime}\right) i \sigma^{i j} u(p, \lambda) .
\end{array}
$$

At tree level the soft function is unity, as well as the Wilson lines in the collinear correlator. Thus we simply have

$$
\left.W_{\lambda \lambda^{\prime}}^{\left[\gamma^{+}\right], q}\right|_{\mathrm{LO}}=\frac{1}{2 P^{+}} \delta(1-x) \delta^{(2)}\left(\bar{k}_{\perp}\right) \bar{u}\left(p^{\prime}, \lambda^{\prime}\right) \gamma^{+} u(p, \lambda)+\mathscr{O}\left(\alpha_{s}\right) .
$$

Comparing this result with the general parametrization (1.1), we can identify the various $F_{1, i}$ distributions (using Gordon identities that appear, for instance, in Appendix A of [3]):

$$
\begin{aligned}
F_{1,1}^{q} & =\left(1-\xi^{2}\right) \delta(1-x) \delta^{(2)}\left(\bar{k}_{\perp}\right)+\mathscr{O}\left(\alpha_{s}\right) \\
F_{1,2}^{q} & =\mathscr{O}\left(\alpha_{s}\right) \\
F_{1,3}^{q} & =\frac{1}{2} \delta(1-x) \delta^{(2)}\left(\bar{k}_{\perp}\right)+\mathscr{O}\left(\alpha_{s}\right) \\
F_{1,4}^{q} & =\mathscr{O}\left(\alpha_{s}\right)
\end{aligned}
$$

Let us now consider the NLO corrections, starting from the virtual ones. The soft function has already been calculated with the $\delta$-regulator in [6]. Using the LO result for the unsubtracted matrix element $\phi$ of (3), which is basically $\delta(1-x)$, we can express the NLO contribution of the soft function to the GTMD in (3). It is

$$
\begin{aligned}
& \left.W_{\lambda \lambda^{\prime}}^{\left[\gamma^{+}\right], q}\right|_{S \text { virtual }}= \\
& \frac{1}{2}\left\{\frac{1}{2 P^{+}} \bar{u}\left(p^{\prime}, \lambda^{\prime}\right) \gamma^{+} u(p, \lambda) \delta(1-x) \delta^{(2)}\left(\bar{k}_{\perp}\right) \frac{\alpha_{s} C_{F}}{2 \pi}\left[-\frac{2}{\varepsilon_{\mathrm{UV}}^{2}}+\frac{2}{\varepsilon_{\mathrm{UV}}} \ln \frac{\delta^{2}}{\mu^{2}}-\ln ^{2} \frac{\delta^{2}}{\mu^{2}}+\frac{\pi^{2}}{2}\right]\right\}
\end{aligned}
$$

where the overall factor of $1 / 2$ comes from the square root of $S$ in (3). One can already notice the term of mixed UV-RD divergences $\frac{1}{\varepsilon_{\mathrm{UV}}} \ln \delta$. This term exemplifies one form through which the problematic feature of RDs is manifested, namely their entanglement with UV divergences.

The virtual gluon contribution from the collinear matrix element $\phi$ is

$$
\begin{aligned}
& \left.W_{\lambda \lambda^{\prime}}^{\left[\gamma^{+}\right], q}\right|_{\phi \text { virtual }}=\frac{1}{2 P^{+}} \bar{u}\left(p^{\prime}, \lambda^{\prime}\right) \gamma^{+} u(p, \lambda) \delta(1-x) \delta^{(2)}\left(\bar{k}_{\perp}\right) \frac{\alpha_{s} C_{F}}{2 \pi}\left[\frac{2}{\varepsilon_{\mathrm{UV}}} \ln \frac{\delta}{P^{+} \sqrt{1-\xi^{2}}}+\frac{3}{2 \varepsilon_{\mathrm{UV}}}\right. \\
& \left.\quad-\frac{3}{2} \ln \frac{\tilde{\Delta}}{\mu^{2}}-2 \ln \frac{\tilde{\Delta}}{\mu^{2}} \ln \frac{\delta}{P^{+} \sqrt{1-\xi^{2}}}-\frac{1}{2} \ln ^{2} \frac{\delta}{P^{+}(1+\xi)}-\frac{1}{2} \ln ^{2} \frac{\delta}{P^{+}(1-\xi)}+\frac{7}{4}+\frac{5}{12} \pi^{2}+i \pi \ln \frac{1-\xi}{1+\xi}\right]
\end{aligned}
$$

\footnotetext{
${ }^{2}$ Strictly speaking, the variables $p, p^{\prime}, P, \lambda, \lambda^{\prime}$ refer to partonic variables in all the results below. However we use the same symbols as for hadronic variables.
} 
where the last result still includes the soft contamination (and thus it is known in the literature as the naive contribution). It also clearly shows the presence of mixed divergences $\frac{1}{\varepsilon_{\mathrm{UV}}} \ln \delta$.

To calculate all the virtual contributions to the GTMD we need to subtract a whole contribution of the soft function, thus obtaining the pure collinear, and then add half of it as dictated by (3). We thus end up subtracting (1.5) from (1.6). The result is

$$
\begin{aligned}
& \left.W_{\lambda \lambda^{\prime}}^{\left[\gamma^{+}\right], q}\right|_{\text {virtual }}= \\
& \frac{1}{2 P^{+}} \bar{u}\left(p^{\prime}, \lambda^{\prime}\right) \gamma^{+} u(p, \lambda) \delta(1-x) \delta^{(2)}\left(\bar{k}_{\perp}\right) \frac{\alpha_{s} C_{F}}{2 \pi}\left[\frac{1}{\varepsilon_{\mathrm{UV}}^{2}}+\frac{1}{\varepsilon_{\mathrm{UV}}}\left(\frac{3}{2}+\ln \frac{\mu^{2}}{Q^{2}\left(1-\xi^{2}\right)}\right)-\frac{3}{2} \ln \frac{\tilde{\Delta}}{\mu^{2}}\right. \\
& \left.-\frac{1}{2} \ln ^{2} \frac{\tilde{\Delta}^{2}}{\mu^{2} Q^{2}}+\ln ^{2} \frac{\tilde{\Delta}}{\mu^{2}}+\ln \frac{\tilde{\Delta}^{2}}{\mu^{2} Q^{2}} \ln \left(1-\xi^{2}\right)-\frac{1}{2} \ln ^{2}(1+\xi)-\frac{1}{2} \ln ^{2}(1-\xi)+\frac{7}{4}+\frac{\pi^{2}}{6}+i \pi \ln \frac{1-\xi}{1+\xi}\right],
\end{aligned}
$$

where $Q^{2}=P^{+} P^{-}$, and without loss of generality we have used the relation $\tilde{\Delta} \equiv Q \delta$ with $Q=P^{+}=$ $P^{-}$to simplify the logarithmic structure. It is clear that this result is free from mixed UV-RDs, as anticipated. Similar conclusions apply to real-gluon emission diagrams: when the contributions from the collinear and soft matrix elements are properly combined, RDs cancel completely.

To conclude, we emphasize that the inclusion of the soft function in the definition of GTMDs completely cancels spurious rapidity divergences and makes them well-defined hadronic quantities, with a proper evolution (discussed below) and all the properties that one would expect to have in such objects.

\section{Evolution of GTMDs}

Given that GTMDs and TMDs are defined through the same bi-local operator, and the same soft function, both have identical evolution. To start with, the anomalous dimension $\gamma_{W}^{j}$ (for $j=$ $q, g$ ) controls the evolution in $\mu$ :

$$
\frac{d}{d \ln \mu} \ln \tilde{W}^{j}\left(b_{T} ; \mu, Q^{2}\right)=\gamma_{W}^{j}\left(\alpha_{s}(\mu), \ln \frac{Q^{2}\left(1-\xi^{2}\right)}{\mu^{2}}\right),
$$

where $\tilde{W}^{q}$ represents the Fourier transform of any of the 16 quark GTMDs that parametrize $W_{\lambda \lambda^{\prime}}^{[\Gamma], q}$ [3], with $b_{T}$ the conjugate variable of $\bar{k}_{T}$ (see [4] for gluon case). $\gamma_{W}^{j}$ has the same functional form as the anomalous dimension of the TMDs (see e.g. [24] for the quark case and [8] for the gluon case),

$$
\gamma_{W}^{j}\left(\alpha_{s}(\mu), \ln \frac{Q^{2}\left(1-\xi^{2}\right)}{\mu^{2}}\right)=-\Gamma_{\text {cusp }}^{j}\left(\alpha_{s}(\mu)\right) \ln \frac{Q^{2}\left(1-\xi^{2}\right)}{\mu^{2}}-\gamma^{j}\left(\alpha_{s}(\mu)\right)
$$

and it is currently known up to third order in $\alpha_{s}$. On the other hand, the evolution in $Q^{2}$ is given by

$$
\frac{d}{d \ln Q^{2}} \ln \tilde{W}^{j}\left(b_{T} ; \mu, Q^{2}\right)=-D^{j}\left(b_{T} ; \mu\right)
$$


where the $D^{j}$ function is the same as for the TMDs, since it is obtained from the contribution of the soft function, which is the same. See $[24,13,8,25]$ for the role of the $D^{j}$ term in the evolution of TMDs.

The complete evolution from a given initial scales $\left(\mu_{0}, Q_{0}^{2}\right)$ to some final scales $\left(\mu, Q^{2}\right)$ is given by combining the evolution in $\mu$ and $Q^{2}$ :

$$
\tilde{W}^{j}\left(b_{T} ; \mu, Q^{2}\right)=R^{j}\left(\xi, b_{T} ; \mu, Q^{2}, \mu_{0}, Q_{0}^{2}\right) \tilde{W}^{j}\left(b_{T} ; \mu_{0}, Q_{0}^{2}\right),
$$

where the evolution kernel $R^{j}$ is

$$
R^{j}\left(\xi, b_{T} ; \mu, Q^{2}, \mu_{0}, Q_{0}^{2}\right)=\left(\frac{Q^{2}}{Q_{0}^{2}}\right)^{-D^{j}\left(b_{T} ; \mu_{0}\right)} \exp \left[\int_{\mu_{0}}^{\mu} \frac{d \hat{\mu}}{\hat{\mu}} \gamma_{W}^{j}\left(\alpha_{s}(\hat{\mu}), \ln \frac{Q^{2}\left(1-\xi^{2}\right)}{\hat{\mu}^{2}}\right)\right] .
$$

The $D^{j}$ term needs to be parametrized at large $b_{T}$ tail with some non-perturbative model, but it is worth noticing that this model has to be the same as the one for TMDs, since the soft function that enters the definitions of both TMDs and GTMDs is the same.

\section{Conclusions}

In [1] we have considered the current formulation of generalized transverse momentum dependent distributions (GTMDs), and argued that they are ill-defined. This observation was supported by a first-order calculation in perturbative QCD, which explicitly showed the appearance of the anticipated spurious rapidity divergences, and their cancellation once the proper definition is considered, by the inclusion of a non-trivial soft factor. With the proper definition of the GTMDs at hand, we managed to obtain their evolution kernel, which is universal and spin independent, and whose non-perturbative contribution is exactly the same as the one that drives the evolution of TMDs, since in both cases we have the same soft factor.

\section{References}

[1] M. G. Echevarria, A. Idilbi, K. Kanazawa, C. Lorcé, A. Metz, B. Pasquini and M. Schlegel, Phys. Lett. B 759 (2016) 336 [arXiv:1602.06953 [hep-ph]].

[2] S. Meissner, A. Metz, M. Schlegel and K. Goeke, JHEP 0808 (2008) 038 [arXiv:0805.3165 [hep-ph]].

[3] S. Meissner, A. Metz and M. Schlegel, JHEP 0908 (2009) 056 [arXiv:0906.5323 [hep-ph]].

[4] C. Lorcé and B. Pasquini, JHEP 1309 (2013) 138 [arXiv:1307.4497 [hep-ph]].

[5] J. C. Collins, Foundations of Perturbative QCD (Cambridge University Press, Cambridge, 2011).

[6] M. G. Echevarria, A. Idilbi and I. Scimemi, JHEP 1207 (2012) 002 [arXiv:1111.4996 [hep-ph]].

[7] M. G. Echevarría, A. Idilbi and I. Scimemi, Phys. Lett. B 726 (2013) 795 [arXiv:1211.1947 [hep-ph]].

[8] M. G. Echevarria, T. Kasemets, P. J. Mulders and C. Pisano, JHEP 1507 (2015) 158 [arXiv:1502.05354 [hep-ph]].

[9] A. V. Belitsky, X. Ji and F. Yuan, Nucl. Phys. B 656 (2003) 165 [hep-ph/0208038].

[10] A. Idilbi and I. Scimemi, Phys. Lett. B 695 (2011) 463 [arXiv:1009.2776 [hep-ph]]. 
[11] M. Garcia-Echevarria, A. Idilbi and I. Scimemi, Phys. Rev. D 84 (2011) 011502 [arXiv:1104.0686 [hep-ph]].

[12] M. G. Echevarria, A. Idilbi and I. Scimemi, Phys. Rev. D 90 (2014) no.1, 014003 [arXiv:1402.0869 [hep-ph]].

[13] M. G. Echevarria, I. Scimemi and A. Vladimirov, Phys. Rev. D 93 (2016) no.5, 054004 [arXiv:1511.05590 [hep-ph]].

[14] A. V. Manohar and I. W. Stewart, Phys. Rev. D 76 (2007) 074002 [hep-ph/0605001].

[15] C. Lorce and B. Pasquini, Phys. Rev. D 84 (2011) 014015 [arXiv:1106.0139 [hep-ph]].

[16] C. Lorce, B. Pasquini, X. Xiong and F. Yuan, Phys. Rev. D 85 (2012) 114006 [arXiv:1111.4827 [hep-ph]].

[17] Y. Hatta, Phys. Lett. B 708 (2012) 186 [arXiv:1111.3547 [hep-ph]].

[18] P. Hagler, A. Mukherjee and A. Schafer, Phys. Lett. B 582 (2004) 55 [hep-ph/0310136].

[19] K. Kanazawa, C. Lorcé, A. Metz, B. Pasquini and M. Schlegel, Phys. Rev. D 90 (2014) no.1, 014028 [arXiv:1403.5226 [hep-ph]].

[20] A. Rajan, A. Courtoy, M. Engelhardt and S. Liuti, Phys. Rev. D 94 (2016) no.3, 034041 [arXiv:1601.06117 [hep-ph]].

[21] Y. Hatta, B. W. Xiao and F. Yuan, Phys. Rev. Lett. 116 (2016) no.20, 202301 [arXiv:1601.01585 [hep-ph]].

[22] X. Ji, F. Yuan and Y. Zhao, arXiv:1612.02438 [hep-ph].

[23] Y. Hatta, Y. Nakagawa, F. Yuan and Y. Zhao, arXiv:1612.02445 [hep-ph].

[24] M. G. Echevarria, A. Idilbi, A. Schäfer and I. Scimemi, Eur. Phys. J. C 73 (2013) no.12, 2636 [arXiv:1208.1281 [hep-ph]].

[25] A. A. Vladimirov, arXiv:1610.05791 [hep-ph]. 